\title{
A!
}

This is an electronic reprint of the original article.

This reprint may differ from the original in pagination and typographic detail.

Zuo, Yonggang; Yu, Wentao; Liu, Can; Cheng, Xu; Qiao, Ruixi; Liang, Jing; Zhou, Xu; Wang, Jinhuan; Wu, Muhong; Zhao, Yun; Gao, Peng; Wu, Shiwei; Sun, Zhipei; Liu, Kaihui; Bai, Xuedong; Liu, Zhongfan

\section{Optical fibres with embedded two-dimensional materials for ultrahigh nonlinearity}

Published in:

Nature Nanotechnology

DOI:

10.1038/s41565-020-0770-x

Published: 01/12/2020

Document Version

Peer reviewed version

Please cite the original version:

Zuo, Y., Yu, W., Liu, C., Cheng, X., Qiao, R., Liang, J., Zhou, X., Wang, J., Wu, M., Zhao, Y., Gao, P., Wu, S., Sun, Z., Liu, K., Bai, X., \& Liu, Z. (2020). Optical fibres with embedded two-dimensional materials for ultrahigh nonlinearity. Nature Nanotechnology, 15(12), 987-991. https://doi.org/10.1038/s41565-020-0770-x

This material is protected by copyright and other intellectual property rights, and duplication or sale of all or part of any of the repository collections is not permitted, except that material may be duplicated by you for your research use or educational purposes in electronic or print form. You must obtain permission for any other use. Electronic or print copies may not be offered, whether for sale or otherwise to anyone who is not an authorised user. 


\section{Optical fibres with embedded two-dimensional materials for ultrahigh nonlinearity}

Yonggang Zuo ${ }^{1,2 \#}$, Wentao $\mathrm{Yu}^{2 \#}, \mathrm{Can}_{\mathrm{Liu}}^{2 \#}, \mathrm{Xu} \mathrm{Cheng}^{2}$, Ruixi Qiao ${ }^{3}$, Jing Liang ${ }^{2}, \mathrm{Xu} \mathrm{Zhou}^{2}$, Jinhuan Wang ${ }^{4}$, Muhong $\mathrm{Wu}^{3}$, Yun $\mathrm{Zhao}^{4}$, Peng $\mathrm{Gao}^{3}$, Shiwei $\mathrm{Wu}^{5}$, Zhipei Sun ${ }^{6}$, Kaihui Liu ${ }^{2,3 *}$, Xuedong Bai ${ }^{1 *}$, Zhongfan $\mathrm{Liu}^{7,8 *}$

${ }^{1}$ Beijing National Laboratory for Condensed Matter Physics, Institute of Physics, Chinese Academy of Sciences, Beijing 100190, China

2 State Key Laboratory for Mesoscopic Physics, Frontiers Science Centre for Nanooptoelectronics, School of Physics, Peking University, Beijing 100871, China

${ }^{3}$ International Centre for Quantum Materials, Collaborative Innovation Centre of Quantum Matter, Beijing 100871, China

4 School of Chemistry and Chemical Engineering, Beijing Institute of Technology, Beijing 100081, China

${ }^{5}$ State Key Laboratory of Surface Physics, Department of Physics, Fudan University, Shanghai 200433, China

6 Department of Electronics and Nanoengineering and QTF Centre of Excellence, Aalto University, Aalto, Finland

${ }^{7}$ Centre for Nanochemistry, College of Chemistry and Molecular Engineering, Peking University, Beijing 100871, China

${ }^{8}$ Beijing Graphene Institute (BGI), Beijing 100095, China

\# These authors contributed equally to this work

* Correspondence: khliu@pku.edu.cn, xdbai@iphy.ac.cn, zfliu@pku.edu.cn 
Nonlinear optical fibres have been employed for a vast number of applications, including optical frequency conversion, ultrafast laser and optical communication ${ }^{1-4}$. In the current manufacture technologies, the nonlinearity is realized by nonlinear material injection into fibres $^{5-7}$ or the fabrication of microstructure fibre ${ }^{8-10}$. Both strategies, however, suffer from either low optical nonlinearity or poor designing flexibility. Here we report direct growth of $\mathrm{MoS}_{2}$, a highly nonlinear two-dimensional (2D) material ${ }^{11}$, onto the internal walls of a structured $\mathrm{SiO}_{2}$ optical fibre. The growth is realized via two-step chemical vapour deposition (CVD) method, in which a solid precursor is pre-deposited to guarantee a homogeneous feedstock prior to a uniform 2D material growth along the whole fibre walls. Thus fabricated, a 25-cm-long fibre shows that both the SHG and THG (second and third harmonic generation) can be enhanced by $\sim 300$ times compared to monolayer $\mathrm{MoS}_{2} /$ silica. Propagation losses remain $\sim 0.1 \mathrm{~dB} / \mathrm{cm}$ for a wide frequency range. In addition, we demonstrate an all-fibre mode-locked laser ( $\sim 6 \mathrm{~mW}$ output, $\sim 500$ fs pulse width and $\sim 41$ MHz repetition rate) by integrating the $2 \mathrm{D}$ material embedded optical fibre as a saturable absorber. Initial tests show that our fabrication strategy is applicable to other transition metal dichalcogenides (TMDs), making these embedded fibres versatile for the several allfibre nonlinear optics and optoelectronics applications.

The superiority of 2D materials on nonlinear optical fibres lies in that the atomically-thin layer won't destroy the high-quality waveguide modes in optical fibres and the enhanced light2D material interaction within fibres can produce ultrahigh nonlinear optical responses ${ }^{12-14}$. Previously, 2D materials were mainly attached to optical fibre facets by transfer techniques, unfortunately suffering the distortion of propagation capacity, the short light-material interaction length and the difficulty of batch manufacture ${ }^{15-17}$. Thanks to the great progress in 2D material growth, it is now possible to directly fabricate graphene optical fibre, wherein the feedstock is of gas phase and therefore relatively easy to diffuse into the narrow holes for homogeneous growth ${ }^{18}$. However, in TMD embedded optical fibre growth, the feedstocks are typically solid precursors 
and thus very difficult to transfer into the fibre holes effectively and homogeneously ${ }^{19-22}$.

To break this dilemma, the key design in our method lies in the pre-deposition step, as shown in Fig. 1a, Mo sources were filled by capillarity of sodium molybdate $\left(\mathrm{Na}_{2} \mathrm{MoO}_{4}\right)$ aqueous solution into the holes of fibres. After that, the fibre was loaded into a CVD furnace to bake for 30 min at a low temperature of $110{ }^{\circ} \mathrm{C}$ for dewetting, and the $\mathrm{Na}_{2} \mathrm{MoO}_{4}$ precursors would gathered into clusters and coated randomly onto the inner fibre walls, serving as a storage for the Mo sources (Fig. 1a, middle panel). And then at a high growth temperature of $\sim 820^{\circ} \mathrm{C}, \mathrm{Na}_{2} \mathrm{MoO}_{4}$ clusters on the hole walls would transform into liquid droplets and continuously release Mo precursors by vapourization in the confined narrow space of fibre holes ${ }^{23}$. The $\mathrm{S}$ vapour was brought in by argon gas from the upstream via a low-pressure pump and then mixed with $\mathrm{Na}_{2} \mathrm{MoO}_{4}$ vapour uniformly for following homogenous $\mathrm{MoS}_{2}$ growth (Fig. 1a, right panel)

In our two-step growth method, the $\mathrm{MoS}_{2}$ coverage and thickness can be facilely tuned by the concentration $c$ of $\mathrm{Na}_{2} \mathrm{MoO}_{4}$ in the solution. When we increased the solute concentration from 1,4 to $8 \mathrm{mg} / \mathrm{mL}$, the $\mathrm{MoS}_{2}$ gradually enlarged from $40 \mu \mathrm{m}$ triangles (Fig. $1 \mathrm{~b}$ ), $250 \mu \mathrm{m}$ triangles (Fig. 1c) to continuous film (Fig. 1d). Further increase of $\mathrm{Na}_{2} \mathrm{MoO}_{4}$ concentration would lead to the growth of few-layered $\mathrm{MoS}_{2}$ (Supplementary Figs. 1 and 2). Under our optimized conditions, we can readily realize homogenous $\mathrm{MoS}_{2}$ growth onto the hole walls of a long optical fibre up to $25 \mathrm{~cm}$ (Supplementary Fig. 3). We have also carried out controlled experiments, in which we didn't add the first step of pre-deposition of Mo precursor onto the hole walls of fibres but directly send $\mathrm{MoO}_{3}$ from outside of the fibres as in prevailing growth methods ${ }^{24}$, and found that only few distributed $\mathrm{MoS}_{2}$ islands could be grown (Supplementary Fig. 4). Thus, the predeposition of Mo source in the fibre holes is the key step for the successful growth of large and uniform $\mathrm{MoS}_{2}$ film onto fibres.

The high-quality $\mathrm{MoS}_{2}$ film grown in the confined space of fibre holes is comparable to that grown on flat substrates in the free space, which is fully confirmed by photoluminescence (PL) 
mapping (Supplementary Fig. 5) of individual triangular domain. These triangles are of singlecrystal domains, as being proved by the parallel polarization SHG patterns at different positions of single domains (Fig. 1f). We further etched the fused silica fibre away and transferred the $\mathrm{MoS}_{2}$ film onto a hollow grid for scanning transmission electron microscopic characterization with atomic resolution. As the tube-like $\mathrm{MoS}_{2}$ monolayer collapsed (Supplementary Fig. 6), clear Moiré pattern of $\mathrm{MoS}_{2}$ bilayers was shown without observable defect sites (Fig. 1e), confirming the high quality of $\mathrm{MoS}_{2}$ domains again. Furthermore, the very slight variations of frequency difference between $A_{1 g}$ and $E_{2 g}$ as well as the narrow distribution of full width at half maxima (FWHM) of E2g peak in Raman spectra along the entire 25-cm-long fibre indicate that the asgrown monolayer $\mathrm{MoS}_{2}$ film is also of high uniformity ${ }^{25,26}$ (Fig. 1g).

Our growth method for TMD embedded optical fibre is proved universal for different fibre structures and 2D materials. Two types of commonly used micro-structured optical fibres, i.e. hollow capillary fibres (HCFs) with different hole diameters from $5 \mu \mathrm{m}, 15 \mu \mathrm{m}$ to $50 \mu \mathrm{m}$ (Fig. 2a and Supplementary Fig. 7) and PCFs with air or solid core (Fig. 2b and Supplementary Fig. 8) were used to successfully grow uniform monolayer $\mathrm{MoS}_{2}$ films (Fig. 2c-d). Since the diameter of hole varies in different fibres, the amount of Mo precursor pre-deposited on the inner hole walls was accordingly adjusted by the solute concentration (i.e., the smaller the diameter, the higher the $\mathrm{Na}_{2} \mathrm{MoO}_{4}$ concentration). This growth method is also proved to be applied to fabricate fibres with different TMDs just by varying the feedstocks of transition metals (e.g. $\mathrm{Na}_{2} \mathrm{MoO}_{4}$, $\mathrm{Na}_{2} \mathrm{WO}_{4}$ ) and chalcogen (e.g. S, Se) species with adjusted growth temperature (see Methods). Raman and PL spectra of these obtained homogenous TMD embedded fibres demonstrate the construction of $\mathrm{MoS}_{2}, \mathrm{WS}_{2}, \mathrm{MoSe}_{2}$ as well as alloys such as $\mathrm{MoS}_{\mathrm{x}} \mathrm{Se}_{2-\mathrm{x}}$ embedded optical fibres (Fig. 2e-f).

Due to their intact light propagation mode (Supplementary Fig. 9), strong light-matter interaction, various fibre structures and rich TMD species, the 2D material embedded optical fibres will have ultrahigh optical nonlinearity and plentiful function-designing flexibility, which 
naturally enable variable potential applications in the wide scopes of nonlinear optics. Since the optical nonlinear susceptibility contains two parts, i.e., the real part and imaginary part, the corresponding applications can be divided into two types: (i) the nonlinear wavelength conversion (e.g., harmonic generation, four-wave mixing frequency), which is the typical demonstration of the real part; and (ii) the nonlinear absorption (e.g., saturable absorption, multiphoton absorption), which is the typical demonstration of the imaginary part. By selecting proper fibre structures and 2D material species, the real and imaginary part related applications have both been demonstrated in our TMD embedded fibres.

We demonstrated that the nonlinear optical harmonic generations (SHG and THG shown in Fig. 3a) of the TMD embedded fibres were greatly enhanced compared to that of conventional optical fibres, wherein the SHG and THG were fairly weak and rarely observed before. As for practical nonlinear wavelength conversion, one will utilize the real part of nonlinear susceptibility and simultaneously ensure the excitation and emission of photons within the bandgap of the materials. Since $\mathrm{MoS}_{2}$ monolayer has a direct bandgap at $\sim 670 \mathrm{~nm}$, we chose long wavelengths $(1800 \mathrm{~nm}$ and $2100 \mathrm{~nm})$ to generate SHG and THG (900 nm and $700 \mathrm{~nm})$ with generated photon energies smaller than the bandgap for low propagation loss at both excited and emitted wavelength (Supplementary Fig. 10). As designed, we observed significantly enhanced harmonic generation in monolayer $\mathrm{MoS}_{2}$ hollow optical fibre with the length up to $\sim 25 \mathrm{~cm}$, which are more than 300 times stronger than that from monolayer $\mathrm{MoS}_{2}$ on a flat fused silica substrate (Fig. 3b-c). The square and cubic dependence of the emission intensity on excitation power reveals that the observed signals are respectively from SHG and THG (Fig. 3d).

Another great advance of 2D material embedded optical fibres in the nonlinear wavelength conversion is that they will have higher damage threshold power. Unlike the direct laser focusing on nonlinear materials in free space, light in fibre can fulfill the waveguide core, resulting in a typical larger light-field area. Therefore, the fibre optics can bear much higher power before the damage of the nonlinear medium. Besides, the 2D materials on the walls of fibres are typically 
interacted with the evanescent light, which is often weaker than light in fibre core. Under the current HCF with hollow core size of $\sim 50 \mu \mathrm{m}$ and wall thickness of $\sim 40 \mu \mathrm{m}$, light will propagate steadily in the side wall (an annular fibre core) and output at the fibre end (Supplementary Fig. 11). The damage threshold power in the $\mathrm{MoS}_{2}$ embedded fibre under $800 \mathrm{~nm}$ excited laser (with high power to reach the damage threshold) is about 3 times higher than that on a planar substrate with focal spot of $\sim 10 \mu \mathrm{m}$ (Fig. 3e). Considering the enhancements in both the light-matter interaction and damage threshold power, the harmonic generation output power in monolayer $\mathrm{MoS}_{2}$ embedded optical fibre is three orders of magnitude higher than planar 2D MoS 2 and the energy conversion can reach $10^{-4}$ to $10^{-3}$, which is already high enough for some applications such as on-chip new wavelength generation and all-fibre optical parametric oscillator. We also observed that the nonlinear enhancement shows a general monotonical increase and a saturation behaviour with the fibre length (Fig. 3f). In principle, the SHG or THG signal should have a quadratic dependence on the length ${ }^{27}$, which is indeed the case here for fibre length within $5 \mathrm{~cm}$. However, when the fibre becomes longer, the loss effect caused by the absorption tail of monolayer $\mathrm{MoS}_{2}$ and the phase mismatching between the excitation and SHG/THG lights leads to the deviation from the quadratic increase and the saturation trend shows up.

Furtherly, we utilized the $\mathrm{MoS}_{2}$ embedded photonic crystal fibres as a saturable absorber (SA) in a fibre laser for ultrafast pulse generation, which is a typical example of the imaginary part related applications. The laser structure is similar to the most commonly demonstrated fibre ring cavity, which contains a pump laser diode, an erbium-doped fibre as gain media, and an optical coupler for output (Fig. 4a). The main difference here is that we replaced the conventional free-space SA film by the $\mathrm{MoS}_{2}$ embedded fibre and therefore realized the all-fibre mode-locked laser. As we use the absorptive properties of 2D materials, we purposely grow 6-8 layered $\mathrm{MoS}_{2}$, which has a larger absorption tail at $\sim 1550 \mathrm{~nm}$ than monolayer ${ }^{28}$. This 3-cm-long few-layered $\mathrm{MoS}_{2} \mathrm{PCF}$ with hollow-core honeycomb structures shows a relative low coupling loss of $\sim 1 \mathrm{~dB}$ (Supplementary Fig. 12) and a nonlinear absorption modulation depth $\left(\alpha_{s}\right)$ of $10 \%$ with a 
saturation peak intensity of $0.8 \mathrm{MW} / \mathrm{cm}^{2}$ (Fig. 4b), which are already comparable to conventional semiconductor $\mathrm{SA}^{15}$ (e.g. modulation depth $\sim 5 \%$ with a saturation peak intensity $\sim 2 \mathrm{MW} / \mathrm{cm}^{2}$ in AlAs/GaAs Bragg-mirrors). Then a stretched-pulse passively mode-locked fibre laser was built by dispersion management. The maximum output power of the pulsed laser is $\sim 6 \mathrm{~mW}$ (under continue-wave laser pump power of $600 \mathrm{~mW}$ and room temperature) with $\sim 41 \mathrm{MHz}$ repetition frequency (Fig. 4c), $\sim 19 \mathrm{~nm}$ spectral bandwidth $(\Delta \lambda), 1560 \mathrm{~nm}$ centre wavelength (Fig. 4d) and $\sim 500$ fs pulse duration (Fig. 4e). The sub-picosecond pulse train shows a high signal-to-noise ratio of $\sim 52 \mathrm{~dB}$ (Supplementary Fig. 13). Considering the relatively broad spectral bandwidth $(\sim 19 \mathrm{~nm})$, the pulse duration could be further compressed to $\sim 200$ fs by group velocity dispersion compensation outside the laser cavity if needed for future applications. The superior performance, massive production ability and environmental adaptability of the $\mathrm{MoS}_{2}$ embedded fibre demonstrate its distinct advantages compared with the conventional 2D materials integrated fibre on facets or external surfaces (Supplementary Tables 1 and 2), and indicate that it is now ready for scientific researches and industrial applications in ultrafast lasers.

We predict that the two-step growth method is also applicable to grow other 2D materials beyond TMDs onto optical fibres. For different application purposes, there are extremely rich choices of various 2D materials with different nonlinear properties. In addition, by fabricating well-designed fibre structures to control the group velocity dispersion, one would realize supercontinuum generation. And if the phase matching between excitation light and nonlinear signal was satisfied, one would further increase the nonlinear conversing efficiency even to $\sim 10 \%$ level (Supplementary Fig. 14), which would be comparable to the conventional bulk optical crystals. If so, it will open up a new regime for the design of nonlinear optical materials, which would inspire versatile nonlinear applications, such as sum-/difference- frequency generation, high-harmonic generation, nonlinear parametric amplification and terahertz source, in optical fibres. 


\section{References}

1 Cotter, D. et al. Nonlinear optics for high-speed digital information processing. Science 286, 15231528, (1999).

2 Agrawal, G. P. Nonlinear fiber optics: its history and recent progress. J. Opt. Soc. Am. B 28, A1-A10, (2011).

3 Granzow, N. et al. Supercontinuum generation in chalcogenide-silica step-index fibers. Opt. Express 19, 21003-21010, (2011).

4 Markos, C. et al. Hybrid photonic-crystal fiber. Rev. Mod. Phys. 89, 045003, (2017).

5 Sazio, P. J. et al. Microstructured optical fibers as high-pressure microfluidic reactors. Science 311, 1583-1586, (2006).

6 Abouraddy, A. et al. Towards multimaterial multifunctional fibres that see, hear, sense and communicate. Nat. Mater. 6, 336-347, (2007).

7 Eggleton, B. J., Luther-Davies, B., Richardson, K. Chalcogenide photonics. Nat. Photon. 5, 141-148, (2011).

8 Skryabin, D., Luan, F., Knight, J., Russell, P. S. J. Soliton self-frequency shift cancellation in photonic crystal fibers. Science 301, 1705-1708, (2003).

9 Dudley, J. M., Genty, G., Coen, S. Supercontinuum generation in photonic crystal fiber. Rev. Mod. Phys. 78, 1135-1184, (2006).

10 Dudley, J. M., Taylor, J. R. Ten years of nonlinear optics in photonic crystal fibre. Nat. Photon. 3, 85-90, (2009).

11 Autere, A. et al. Nonlinear optics with 2D layered materials. Adv. Mater. 30, 1705963, (2018).

$12 \mathrm{Li}$, Y. et al. Probing symmetry properties of few-layer $\mathrm{MoS}_{2}$ and h-BN by optical second-harmonic generation. Nano Lett. 13, 3329-3333, (2013).

13 Liu, X., Guo, Q., Qiu, J. Emerging low-dimensional materials for nonlinear optics and ultrafast photonics. Adv. Mater. 29, 1605886, (2017).

14 Liu, H. et al. High-harmonic generation from an atomically thin semiconductor. Nat. Phys. 13, 262265, (2017).

15 Wang, F. et al. Wideband-tuneable, nanotube mode-locked, fibre laser. Nat. Nanotechnol. 3, 738742, (2008).

16 Bao, Q. et al. Broadband graphene polarizer. Nat. Photon. 5, 411-415, (2011).

17 Lee, E. J. et al. Active control of all-fibre graphene devices with electrical gating. Nat. Commun. 6, 6851, (2015).

18 Chen, K. et al. Graphene photonic crystal fibre with strong and tunable light-matter interaction. Nat. Photon. 13, 754-759, (2019).

19 Van Der Zande, A. M. et al. Grains and grain boundaries in highly crystalline monolayer 
molybdenum disulphide. Nat. Mater. 12, 554-561, (2013).

20 Huang, C. et al. Lateral heterojunctions within monolayer $\mathrm{MoSe}_{2}-\mathrm{WSe}_{2}$ semiconductors. Nat. Mater. 13, 1096-1101, (2014).

21 Li, M.-Y. et al. Epitaxial growth of a monolayer $\mathrm{WSe}_{2}-\mathrm{MoS}_{2}$ lateral pn junction with an atomically sharp interface. Science 349, 524-528, (2015).

22 Gao, Y. et al. Large-area synthesis of high-quality and uniform monolayer $\mathrm{WS}_{2}$ on reusable Au foils. Nat. Commun. 6, 8569, (2015).

23 Pistorius, C. W. Phase diagrams of sodium tungstate and sodium molybdate to $45 \mathrm{kbar}$. J. Chem. Phys. 44, 4532-4537, (1966).

$24 \mathrm{Yu}, \mathrm{H}$. et al. Wafer-scale growth and transfer of highly-oriented monolayer $\mathrm{MoS}_{2}$ continuous films. ACS Nano 11, 12001-12007, (2017).

25 Lee, C. et al. Anomalous lattice vibrations of single-and few-layer $\mathrm{MoS}_{2}$. ACS Nano 4, 2695-2700, (2010).

26 Liu, K.-K. et al. Growth of large-area and highly crystalline $\mathrm{MoS}_{2}$ thin layers on insulating substrates. Nano Lett. 12, 1538-1544, (2012).

27 Shen, Y.-R., The Principles of Nonlinear Optics Ch.7 (Wiley, New York, 1984).

28 Zhang, H. et al. Molybdenum disulfide $\left(\mathrm{MoS}_{2}\right)$ as a broadband saturable absorber for ultra-fast photonics. Opt. Express 22, 7249-7260, (2014). 


\section{Methods:}

Pre-deposition of transition metal feedstock into optical fibres. Transition metal sources (Mo or W) were pre-deposited in fibres by capillarity of their corresponding saline solution with optimized concentrations. For monolayer film growth, the amount of metal precursor was tuned according to the hole diameters of different fibres. Typical concentrations are listed as below: $\mathrm{Na}_{2} \mathrm{MoO}_{4}$ of 8, 21 and $45 \mathrm{mg} / \mathrm{mL}$ or $\mathrm{Na}_{2} \mathrm{WO}_{4}$ of 12,30 and $64 \mathrm{mg} / \mathrm{mL}$ for $\mathrm{HCF}$ with core diameter of 50,15 and $5 \mu \mathrm{m}, \mathrm{Na}_{2} \mathrm{MoO}_{4}$ of 32 and $54 \mathrm{mg} / \mathrm{mL}$ or $\mathrm{Na}_{2} \mathrm{WO}_{4}$ of 45 and $72 \mathrm{mg} / \mathrm{mL}$ for PCF with hollow- and solid-core honeycomb structures, respectively. The fibres were then heated to $110{ }^{\circ} \mathrm{C}$ and maintained for $30 \mathrm{~min}$ at argon atmosphere to dry before CVD growth.

Syntheses of TMD embedded optical fibres. The Mo precursor pre-deposited fibres were placed on a quartz plate in the centre of the CVD furnace, and $1 \mathrm{~g}$ sulphur (99\%, Sigma Aldrich) powder was placed at the upstream region outside the heating zone. Then, the chamber of furnace was flushed with argon for inert atmosphere and subsequently ramped up to the optimized growth temperature $\left(\sim 820^{\circ} \mathrm{C}\right.$ for $\mathrm{MoS}_{2}$ growth) with $100 \mathrm{sccm}$ argon. During growth, the temperature for sulphur evaporation was $\sim 120^{\circ} \mathrm{C}$. The entire process was carried out under $\sim 150 \mathrm{~Pa}$ and the growth duration was set as 15-30 minutes. After the growth, the system was naturally cooled down to room temperature. Similar growth conditions were used to synthesize other TMD and alloy fibres, including $\mathrm{MoSe}_{2}, \mathrm{WS}_{2}$ and $\mathrm{MoS}_{\mathrm{x}} \mathrm{Se}_{2-\mathrm{x}}$ except for the different temperatures for chalcogen sublimation and TMD growth. Specifically, sublimation temperatures were respectively $\sim 120^{\circ} \mathrm{C}$ for sulphur and $\sim 220^{\circ} \mathrm{C}$ for selenium, and the growth temperature were respectively $\sim 800^{\circ} \mathrm{C}, \sim 950{ }^{\circ} \mathrm{C}$ and $\sim 820^{\circ} \mathrm{C}$ for $\mathrm{MoSe}_{2}, \mathrm{WS}_{2}$ and $\mathrm{MoS}_{\mathrm{x}} \mathrm{Se}_{2-\mathrm{x}}$ embedded fibres.

Characterization of TMD embedded optical fibres. Optical images were taken with an Olympus BX51M microscope by focusing on the hole walls of the fibres. Raman and PL spectra were collected with a homemade optical system using a $532 \mathrm{~nm}$ laser excitation with power of $\sim 1 \mathrm{~mW}$. Scanning transmission electron microscopic (FEI Titan Themis G2 300) was conducted 
to characterize the atomic structure and scanning electron microscope (Thermal Fisher, Quattro S) was used to characterize the morphology of samples.

Harmonic generation measurements. An optical parameter oscillator (Coherent, Mira-OPO-X) pumped by Ti sapphire oscillator (Coherent, Mira-HP) provided a wavelength tunable excitation laser $(15 \mathrm{~mW}, \sim 150 \mathrm{fs}, 76 \mathrm{MHz})$, and then it was focused into the $\mathrm{MoS}_{2} \mathrm{HCF}$ or on the $\mathrm{MoS}_{2} /$ silica by an objective (Nikon, $10 \mathrm{X}, \mathrm{NA}=0.25$ ). The $\mathrm{SHG}$ and THG generation signals were collected by a spectrograph with a silicon-based CCD (Princeton Instrument, SP2500 and Pylon 400BRX) via a transmission system.

Saturable absorption measurements. A homemade pulsed fibre laser (200 fs, $1564 \mathrm{~nm}, 75.4$ $\mathrm{MHz}$ ) was utilized to measure the power-dependence transmission of $\mathrm{MoS}_{2} \mathrm{PCF}$. The pulsed laser was equally divided using a fibre optics coupler to monitor the power. The input/out single mode fibre (Corning, SM-28e+) was aligned with the 3-cm-long $\mathrm{MoS}_{2}$ PCF (NKT Photonics, HC-1550) by a home-built alignment setup which includes two optical microscopes in different directions and multi-axis stages to ensure accurate alignment.

Implement and characterization of all-fibre mode-locked laser. A laser diode (600 mW, 976 $\mathrm{nm}$ ) was coupled into the fibre ring cavity via a wavelength-division multiplexer as pump source of 60-cm-long erbium-doped fibre (LIEKKI, Er110) as gain medium. An isolator guaranteed that the laser in the fibre ring transferred along one direction. In the case, we employed backward pumping configuration for large output power. 3-cm-long $\mathrm{MoS}_{2} \mathrm{PCF}$ was integrated as SA with a coupler for $20 \%$ output. An additional polarization controller was utilized to optimize the output spectrum and pulse width. The total group velocity dispersion of the fibre ring was $0.0005 \mathrm{ps}^{2}$, consisting of $1 \mathrm{~m}$ single mode fibre, $0.6 \mathrm{~m}$ erbium-doped fibre and $3.4 \mathrm{~m}$ OSF980 (as dispersion compensation fibre) with $-22,+12$ and $+4.5 \mathrm{fs}^{2} / \mathrm{mm}$, respectively. The output spectra and autocorrelation trace were measured by a spectrograph with infrared-CCD (Princeton Instruments, HRS-300SS with 150 grooves/mm grating and NIRvana-640, the resolution of the spectrometer $\sim 1.3 \mathrm{~nm}$ ) and an auto-correlator (APE, Pulsecheck USB-15). The pulse train and 
signal-to-noise ratio were observed by an oscilloscope (Rigol, DS6104) and a radio frequency spectrograph (Rigol, DSA815). Self-starting mode-locked pulse was observed when the pump power was increased to $180 \mathrm{~mW}$.

\section{Data availability}

The authors declare that the data supporting the findings of this study are available within the paper and its Supplementary Information, and also from the authors upon request.

\section{Acknowledgements}

This work was supported by Beijing Natural Science Foundation (JQ19004), the National Natural Science Foundation of China (51991340, 51991342, 51991344 and 51522201), National Key R\&D Program of China (2016YFA0300903 and 2016YFA0300804), Beijing Excellent Talents Training Support (2017000026833ZK11) , Beijing Graphene Innovation Program (Z181100004818003), Beijing Municipal Science \& Technology Commission (Z191100007219005), The Key R\&D Program of Guangdong Province (2019B010931001, 2020B010189001, 2018B010109009 and 2018B030327001), Guangdong Innovative and Entrepreneurial Research Team Program (2016ZT06D348), Bureau of Industry and Information Technology of Shenzhen (Graphene platform 201901161512), the Science, Technology and Innovation Commission of Shenzhen Municipality (KYTDPT20181011104202253), National Equipment Program of China (ZDYZ2015-1), National Postdoctoral Program for Innovative Talents (BX20180013 and BX20190016), the Academy of Finland, the ERC (834742), the European Union's Horizon 2020 research and innovation programme (820423, S2QUIP) and China Postdoctoral Science Foundation (2019M660001, 2019M660280 and 2019M660281). We acknowledge the Electron Microscopy Laboratory in Peking University for the use of electron microscope. 


\section{Author contributions}

Z.L., X.B. and K.L. conceived the experiments and supervised the project. Y.G.Z. and C.L. performed the growth experiments. W.Y. contributed the optical experiments and fibre laser setup. X.C. contributed the theoretical modelling. R.Q., P.G. and X.B. conducted the STEM experiments. J.L., X.Z., J.W., M.W., and Y.Z. conducted the SEM, PL and Raman characterizations. S.W. and Z.S. suggested the optical experiments. All of the authors discussed the results and wrote the manuscript.

\section{Competing interests}

The authors declare no competing interests.

\section{Additional information}

Supplementary information is available for this paper at https://doi.org/****

Correspondence and requests for materials should be addressed to K.L., X.B. or Z.L.

Reprints and permissions information is available at www.nature.com/reprints. 


\section{Figures and captions}

\section{Figure 1 | Two-step growth of high-quality uniform monolayer $\mathrm{MoS}_{2}$ embedded optical}

fibre. a, Schematic diagram of the designed two-step growth method, consisting a pre-deposition of $\mathrm{Na}_{2} \mathrm{MoO}_{4}$ onto the hole walls of fibre by capillary filling and low-temperature drying, and a high-temperature growth afterwards. b-d, The dependence of $\mathrm{MoS}_{2}$ coverage on the $\mathrm{Na}_{2} \mathrm{MoO}_{4}$ aqueous solution concentrations of $1 \mathrm{mg} / \mathrm{mL}$ (b), $4 \mathrm{mg} / \mathrm{mL}$ (c) and $8 \mathrm{mg} / \mathrm{mL}$ (d) respectively. e, Scanning transmission electron microscopic (STEM) image of a collapsed tube-like $\mathrm{MoS}_{2}$ transferred onto a hollow grid, showing the high crystallinity of as-grown $\mathrm{MoS}_{2}$ without detectable defects. f, Parallel polarization second harmonic generation patterns at different positions labelled in (c), revealing the whole $\mathrm{MoS}_{2}$ domain is a large single crystal. g, Statistical frequency differences of $\Delta \omega\left(\mathrm{A}_{1 \mathrm{~g}}-\mathrm{E}_{2 \mathrm{~g}}\right)\left(\right.$ left y-axis) and FWHM values of the $\mathrm{E}_{2 \mathrm{~g}}$ peak (right yaxis) plotted as a function of numbered sites on $\mathrm{MoS}_{2}$ embedded fibre along the whole length of $25 \mathrm{~cm}$. The slight variations prove that the $\mathrm{MoS}_{2}$ film is uniformly grown onto the fibre. 
Figure 2 | 2D material embedded optical fibres with diverse fibre structures and material species. a,b, Optical images of a hollow capillary fibre (HCF) with a core diameter of $\sim 5 \mu \mathrm{m}(\mathrm{a})$ and a photonic crystal fibre (PCF) with hollow-core honeycomb structure (b). c,d, The corresponding side views of HCF (c) and PCF (d) shown in (a) and (b) respectively. Higher contrast was observed after the growth of $\mathrm{MoS}_{2}$ (bottom panels) compared with the bare ones (top panels). e,f, PL (e) and Raman (f) spectra of as-grown $\mathrm{MoSe}_{2}$ (green), $\mathrm{MoS}_{2}$ (dark yellow), $\mathrm{WS}_{2}$ (orange) and $\mathrm{MoS}_{\mathrm{x}} \mathrm{Se}_{2-\mathrm{x}}$ (violet) embedded fibres. The PL and Raman spectra are shifted vertically for clarity. 
Figure 3 | Greatly enhanced harmonic generation in $\mathrm{MoS}_{2}$ embedded hollow capillary fibre (HCF). a, Schematics of second harmonic generation (SHG, 2 $\omega$ ) and third harmonic generation (THG, $3 \omega$ ) in $\mathrm{MoS}_{2} \mathrm{HCF}$. b,c, SHG (b) and THG (c) spectra of MoS $2 \mathrm{HCF}, \mathrm{MoS}_{2}$ on flat fused silica substrate $\left(\mathrm{MoS}_{2} /\right.$ silica) and bare HCF under 1800 and $2100 \mathrm{~nm}$ excitation, respectively. With the strong light-matter interaction in 25-cm-long $\mathrm{MoS}_{2} \mathrm{HCF}$, both the SHG and THG can be enhanced by 300 times compared to monolayer $\mathrm{MoS}_{2} /$ silica. For comparison, no apparent SHG or THG signal can be observed in bare HCF. The SHG and THG spectra are shifted vertically for clarity. d, Excitation power dependence of SHG and THG intensity for $\mathrm{MoS}_{2}$ HCF, showing the as-expected quadratic and cubic law. e, Measured SHG intensity of 12-cmlong $\mathrm{MoS}_{2} \mathrm{HCF}$ and $\mathrm{MoS}_{2} /$ silica as a function of the excitation power under $800 \mathrm{~nm}$ excitation. Benefiting from the larger mode area and evanescent light coupling, the damage threshold of $\mathrm{MoS}_{2} \mathrm{HCF}$ is about three times higher than $\mathrm{MoS}_{2} /$ silica. f, Fibre length-dependent SHG and THG enhancements of $\mathrm{MoS}_{2} \mathrm{HCF}$ with respect to $\mathrm{MoS}_{2} /$ silica, showing a general monotonical increase and a saturation behaviour with the fibre length. 


\section{Figure 4 | Ultrafast laser based on $\mathrm{MoS}_{2}$ embedded photonic crystal fibre (PCF). a,}

Schematic diagram of all-fibre mode-locked laser with $\mathrm{MoS}_{2}$ embedded fibre as a saturable absorber. The optical components consist of a laser diode (LD), a wavelength-division multiplexer (WDM), single mode fibre (SMF), dispersion compensation fibre (DCF), erbiumdoped fibre (EDF), an isolator (ISO) and a polarization controller (PC). b, Transmission measurement of the $\mathrm{MoS}_{2}$ PCF with a nonlinear absorption modulation depth $\left(\alpha_{s}\right)$ of $\sim 10 \%$ and a saturation peak intensity of $0.8 \mathrm{MW} / \mathrm{cm}^{2}$. The solid curve is fitted according to the experimental data (circle symbols). c, Output pulse train with $\sim 24 \mathrm{~ns}$ interval ( $\sim 41 \mathrm{MHz}$ repetition rate). d, Spectrum of the output laser with a $\sim 19 \mathrm{~nm}$ spectral bandwidth $(\Delta \lambda)$. e, Autocorrelation trace with FWHM $(\Delta \tau)$ of $\sim 720$ fs fitted by a Gaussian function, corresponding to a pulse duration of $\sim 500$ fs. 

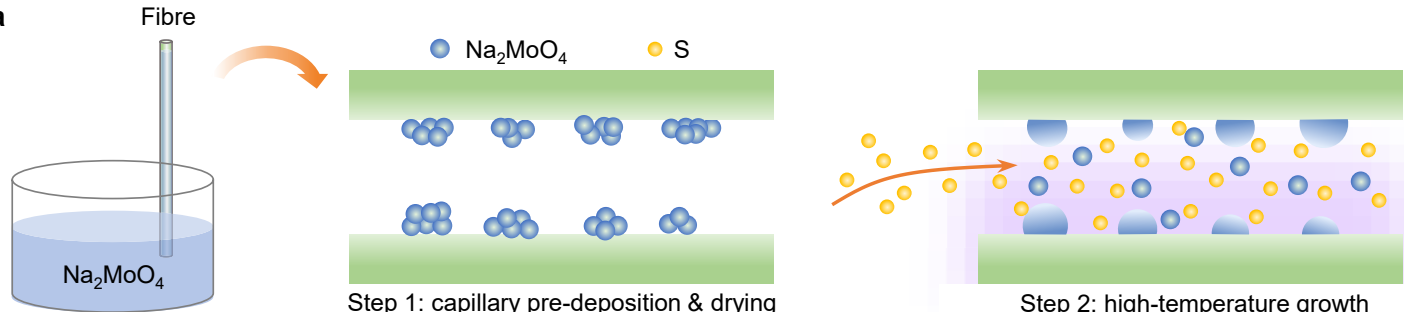

Step 1: capillary pre-deposition \& drying

Step 2: high-temperature growth
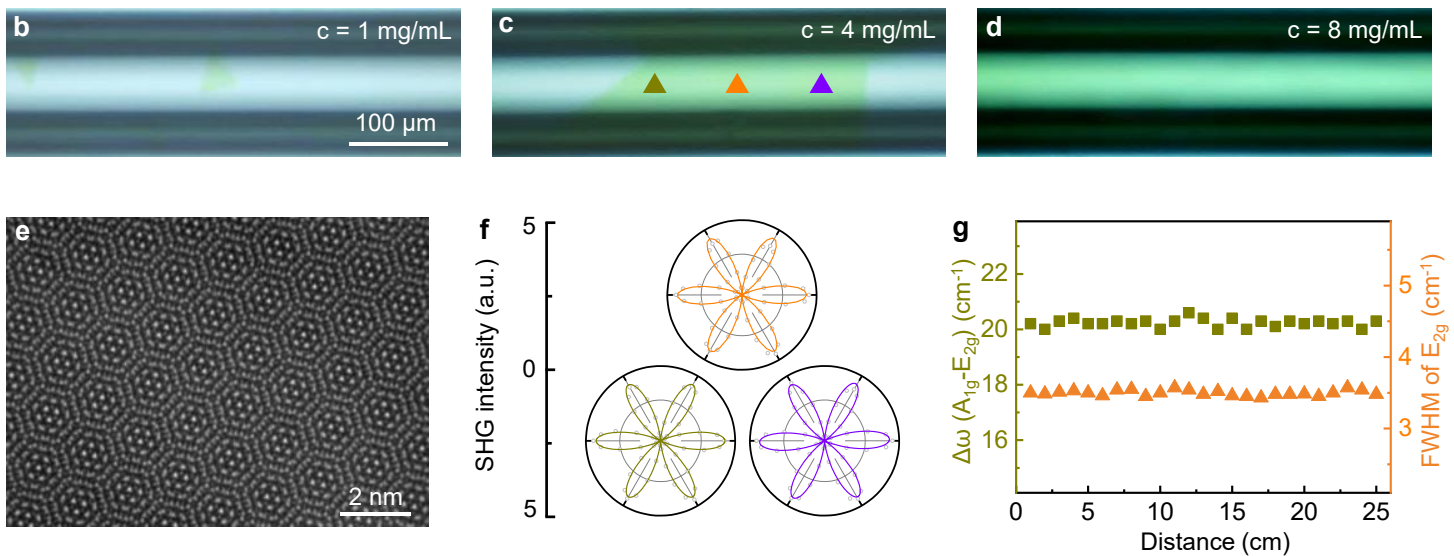

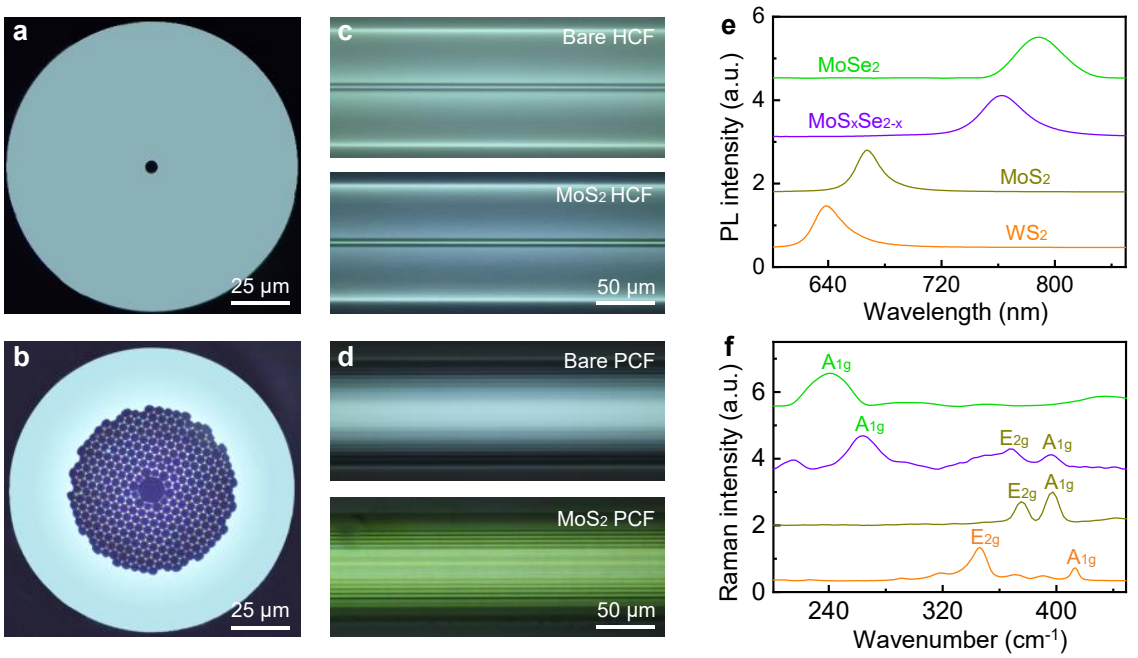

Figure 2 
a

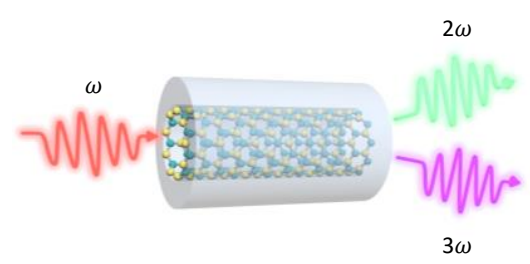

$3 \omega$

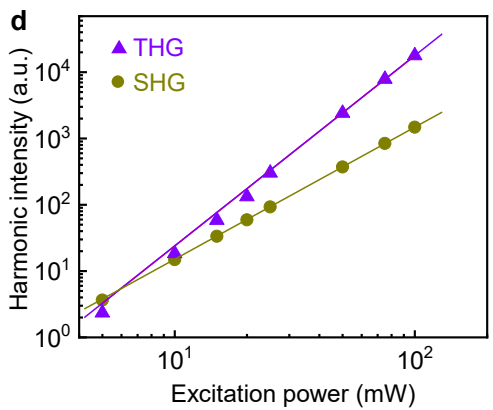

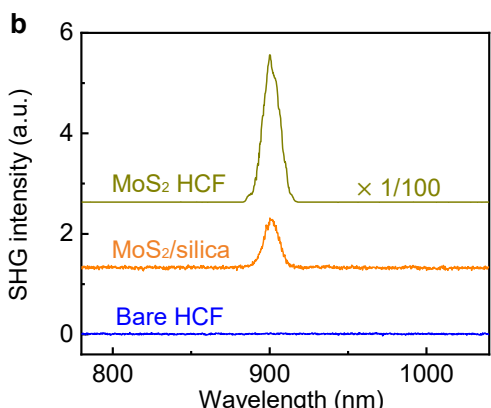
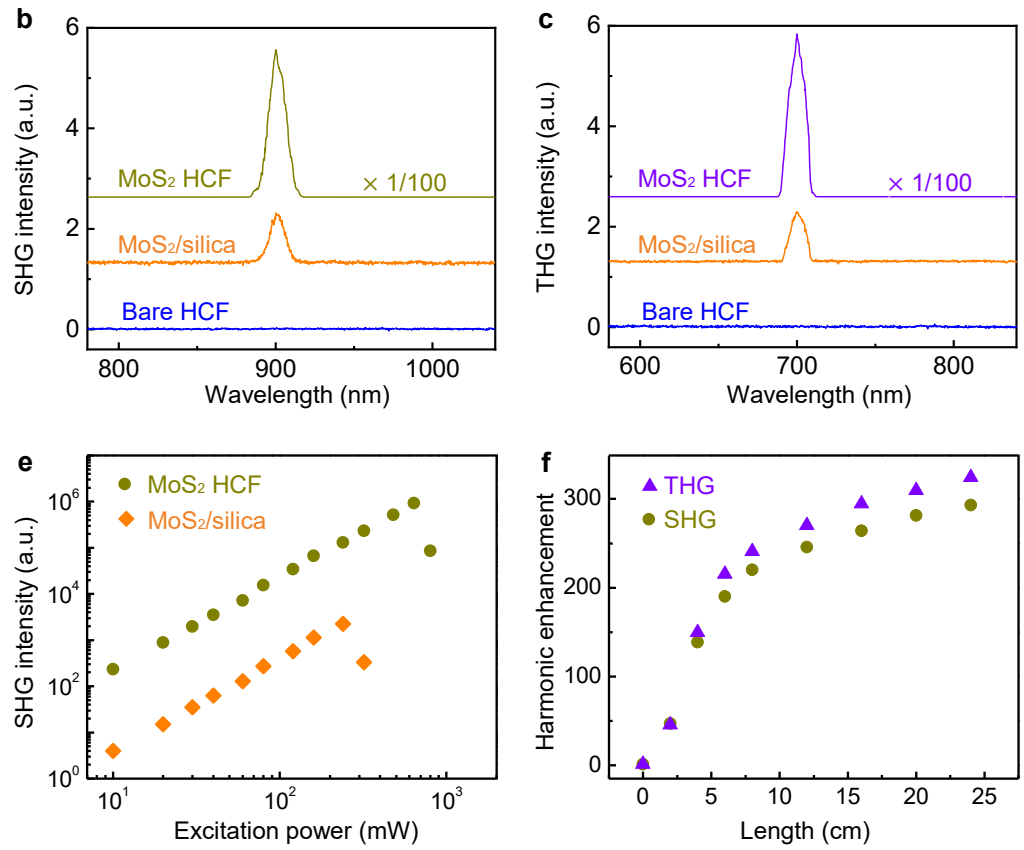
a

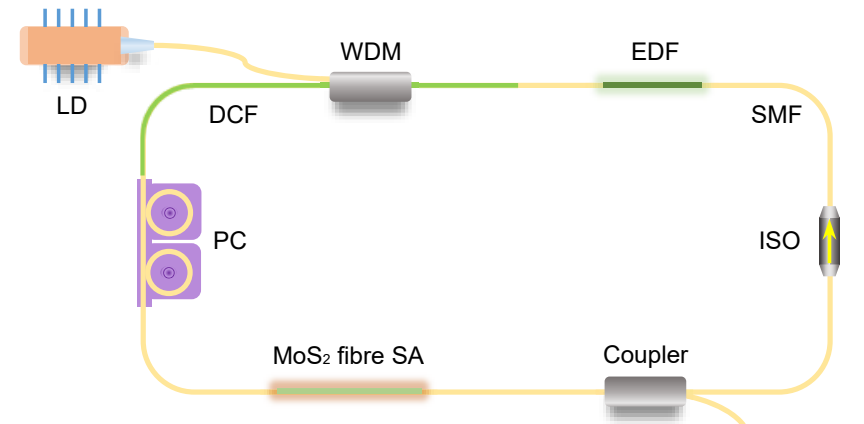

$20 \%$ output
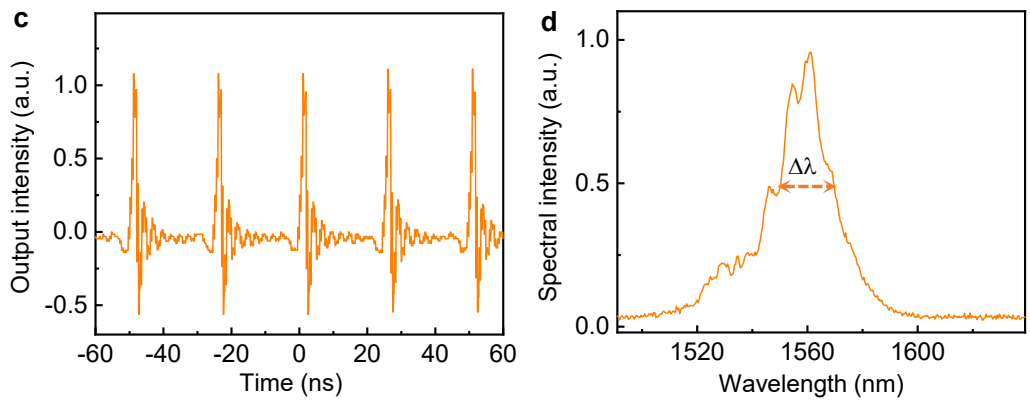
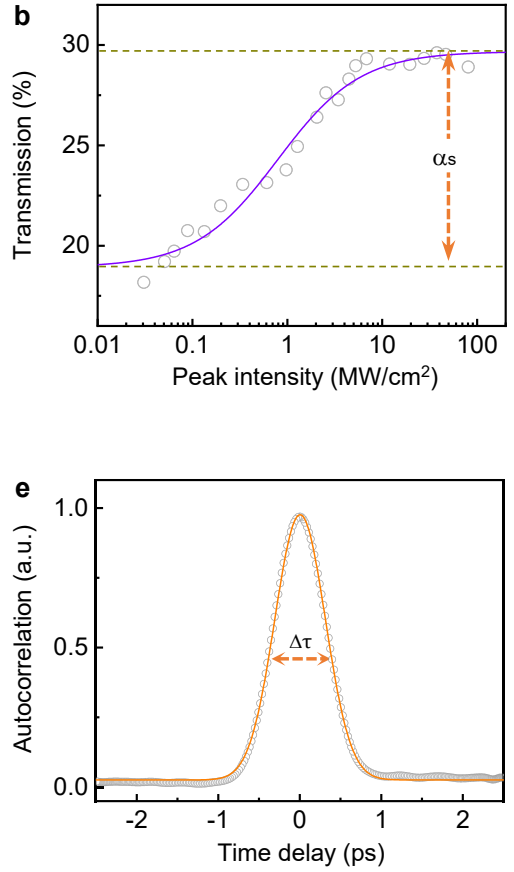